\title{
Nonequilibrium phase transition in the Kinetic Ising model: Is transition point the maximum lossy point?
}

\author{
Muktish Acharyya ${ }^{+}$ \\ Institute for Theoretical Physics \\ University of Cologne, 50923 Cologne, Germany
}

(July 13, 2021)

\begin{abstract}
The nonequilibrium dynamic phase transition, in the kinetic Ising model in presence of an oscillating magnetic field, has been studied both by Monte Carlo simulation (in two dimension) and by solving the meanfield dynamical equation of motion for the average magnetization. The temperature variations of hysteretic loss (loop area) and the dynamic correlation have been studied near the transition point. The transition point has been identified as the minimum-correlation point. The hysteretic loss becomes maximum above the transition point. An analytical formulation has been developed to analyse the simulation results. A general relationship among hysteresis loop area, dynamic order parameter and dynamic correlation has also been developed.
\end{abstract}

PACS number(s): 05.50.+q

\section{INTRODUCTION}

The dynamics of magnetization reversal in simple ferromagnetic systems has recently attracted considerable scientific interest to study the nonequilibrium responses. In this regard, the dynamical responses of the Ising system in presence of an oscillating magnetic field have been studied extensively [1] 6]. The dynamical hysteretic response [1] 3 ] and the nonequilibrium dynamical phase transition [4] 9] are two main subjects of interest to study the dynamic responses of the kinetic Ising model in presence of an oscillating magnetic field.

Tome and Oliviera [4] first studied the dynamic transition by solving the mean field (MF) dynamic equation of motion (for the average magnetisation) of the kinetic Ising model in presence of a sinusoidally oscillating magnetic field. By defining the order parameter as the time averaged magnetisation over a full cycle of the oscillating magnetic field, they showed that the order parameter vanishes depending upon the value of the temperature and the amplitude of the oscillating field. In the field amplitude and temperature plane they have drawn a phase boundary separating dynamic ordered (nonzero value of order parameter) and disordered (order parameter vanishes) phase. They [4] have also observed and located a tricritical point (TCP), (separating the nature (discontinuous/continuous) of the transition) on the phase boundary line.

Since, this transition exists even in the static (zero frequency) limit such a transition, observed [4] from the solution of mean field dynamical equation, can not be dynamic in true sense. This is because, for the field amplitude less than the coercive field (at temperature less than the static ferro-para transition temperature), the response magnetisation varies periodically but asymmetrically even in the zero frequency limit; the system remains locked to one well of the free energy and cannot go to the other one, in the absence of noise or fluctuation. On the other hand, in presence of thermal fluctuations, in the static limit, the system can go from one well to another via the formation of nucleating droplets. Vanishingly small field is required to push the system from one to other well. Consequently, the dynamic phase boundary collapses, in the presence of thermal fluctuations.

To study the true dynamic phase transition (which should disappear in the static limit) one has to consider the effect of thermal fluctuations. In this regard, Lo and Pelcovits [5] first attempted to study the dynamic nature of this phase transition (incorporating the effect of fluctuation) in the kinetic Ising model by Monte Carlo (MC) simulation. However, they [5] have not reported any precise phase boundary. Acharyya and Chakrabarti [6] studied the nonequilibrium dynamic phase transition in the kinetic Ising model in presence of oscillating magnetic field by extensive MC simulation. They [6] have drawn the phase boundary and located a tricritical point (as observed) on the boundary. It has been also observed [6] that this dynamic phase transition is associated with the breaking of the symmetry of the dynamic hysteresis $(m-h)$ loop. In the dynamically disordered (value of order parameter vanishes) phase the corresponding hysteresis loop is symmetric, and loses its symmetry in the ordered phase (giving nonzero value of dynamic order parameter). They have [6] also studied the temperature variation of the ac susceptibility components near the dynamic transition point. It has been observed [6] that the imaginary or lossy (real) part of the ac susceptibility gives a peak (dip) near the dynamic transition point (where the dynamic order parameter vanishes). It was concluded that this is a possible indication of the thermodynamic nature of this kind of nonequilibrium dynamical phase transition. 
The statistical distribution of dynamic order parameter has been studied by Sides et al [7]. The nature of the distribution changes (from bimodal to unimodal) near the dynamic transition point. They have also observed [7] that the fluctuation of the hysteresis loop area grows and becomes considerably large as one approach the dynamic transition point.

The relaxation behaviour, of the dynamic order parameter, near the transition point (in the disordered phase), has been studied [8] recently by MC simulation and solving meanfield dynamic equation. It has been observed that the relaxation is Debye type and the relaxation time diverges near the transition point. The 'specific heat' and the 'susceptibility' also diverge [9] near the transition point in a similar manner with that of fluctuations of order parameter and fluctuation of energy respectively. These observations [9] (divergences of fluctuations) indirectly supports the earlier facts [7] where the distribution of the dynamic order parameter becomes wider and the fluctuation of hysteresis loop area becomes considerably large near the transition point.

Recently the experimental evidence [10] of dynamic transition has been found. The dynamical symmetry breaking (associated to the dynamic transition) across the transition point of the hysteresis loop, has been observed, in highly anisotropic (Ising like) and ultrathin $\mathrm{Co} / \mathrm{Cu}(001)$ ferromagnetic films by surface magneto-optic Kerr effect, as one passes through the transition point. The dynamical symmetry breaking in the hysteresis loops has also been observed [11] in ultrathin Fe/W(110) film. However, the detailed natures of the dynamic transition and the phase boundary are not yet studied experimentally.

In this communication, the dynamic phase transition has been studied in the kinetic Ising model in presence of a sinusoidally oscillating magnetic field by MC simulation and by solving the mean field dynamical equation of motion for the average magnetization. The temperature variations of the hysteresis loss (or loop area), the dynamic correlation and the phase lag are studied near the dynamic transition point. The paper has been organised as follows: in section II simple analytic forms are given for the loop area, dynamic correlation and dynamic order parameter. In section III a general relationship has been developed among the various dynamical quantities. In section IV the models are introduced and in section $\mathrm{V}$ the numerical results are given. The paper ends with a summary of the work in section VI.

\section{ANALYTIC FORMS OF THE LOOP AREA AND THE DYNAMIC CORRELATION NEAR THE TRANSITION POINT}

The form of the oscillating magnetic field is

$$
h(t)=h_{0} \cos (\omega t)
$$

The dynamic order parameter is defined as

$$
Q=\frac{\omega}{2 \pi} \oint m(t) d t
$$

which is nothing but the time averaged magnetisation over a full cycle of the oscillating magnetic field. The hysteresis loop area is

$$
A=-\oint m d h=h_{0} \omega \oint m(t) \sin (\omega t) d t
$$

which corresponds the energy loss due to the hysteresis. The Dynamic correlation is defined as

$$
C=<m(t) h(t)>-<m(t)><h(t)>
$$

where $\langle.$.$\rangle denotes the time average over the full cycle of the oscillating magnetic field. Since <h(t)>=0$, one can write

$$
C=\frac{\omega}{2 \pi} \oint m(t) h(t) d t=\frac{\omega h_{0}}{2 \pi} \oint m(t) \cos (\omega t) d t .
$$

The dynamic correlation has another physical interpretation. For the cooperatively interacting spin system, this is the negative of the time averaged spin-field interaction energy (per spin) $\left(<E_{f}>=-\frac{\omega}{2 \pi L^{2}} \oint \sum_{i} \sigma_{i} h(t) d t\right)$ over a complete cycle of the oscillating field.

In the dynamically disordered $(Q=0)$ phase and near the transition point, the time series of the magnetisation $(m(t))$ can be approximated as a square wave with a phase lag $\delta$ with the applied sinusoidal magnetic field. 


$$
m(t)=\left\{\begin{array}{rll}
1 & \text { for } & 0<t<\tau / 4+\delta / \omega \\
-1 & \text { for } & \tau / 4+\delta / \omega<t<3 \tau / 4+\delta / \omega \\
1 & \text { for } & 3 \tau / 4+\delta / \omega<t<2 \pi / \omega
\end{array}\right.
$$

where $\tau$ is the time period of the oscillating field and $\delta$ is the phase lag between magnetisation $m(t)$ and the magnetic field $h(t)=h_{0} \cos (\omega t)$. The value of the hysteresis loop area can easily be calculated as

$$
A=4 h_{0} \sin (\delta) .
$$

This form of the loop area was also obtained [6] from the assumption that it is approximately equal to four times the product of coercive field and remanent magnetization (here the remanent magnetisation equal to unity), where the coercive field is identified as $h_{0} \sin (\delta)$ (the change in field during the phase lag). Considering the same form of the magnetisation the dynamic correlation $C$ can also be calculated exactly as

$$
C=\frac{2 h_{0}}{\pi} \cos (\delta) .
$$

From the above forms of $A$ and $C$ it can be written as

$$
\frac{A^{2}}{\left(4 h_{0}\right)^{2}}+\frac{C^{2}}{\left(2 h_{0} / \pi\right)^{2}}=1
$$

The above relation tells that the loop area $A$ and the dynamic correlation $C$ is elliptically related to each other. It may be noted here, that the previously studied ac susceptibility components [6] obey a circular relationship $\left(\chi^{\prime 2}+\chi^{\prime \prime 2}\right.$ $\left.=\left(m_{0} / h_{0}\right)^{2}\right)$, where $m_{0}$ is the amplitude of the magnetization.

The ordered region $(Q \neq 0)$ can be approximated by considering the following form of the magnetization

$$
m(t)=\left\{\begin{array}{rll}
1 & \text { for } & 0<t<\tau / 4+\delta / \omega \\
1-m_{r} & \text { for } & \tau / 4+\delta / \omega<t<3 \tau / 4+\delta / \omega \\
1 & \text { for } & 3 \tau / 4+\delta / \omega<t<2 \pi / \omega .
\end{array}\right.
$$

In the above simplified approximation, it was considered that the magnetisation can not jump to the other well, however the value of initial magnetisation is reduced by the amount $m_{r}$. In the real situation it has been observed that this well is not fully square (as assumed above in the form of $m(t)$ ), it has a cusp like (or parabolic) shape. For $m_{r}=2$, the above functional form of $m(t)$ will take the form of 2.5 and one can get the disordered $(Q=0)$ phase. Taking the above form of magnetisation the dynamic order parameter $Q$ can be calculated as $Q=\left(2-m_{r}\right) / 2$. It may be noted that, in this simplified approximation the dynamic order parameter $Q$ is independent of phase lag $\delta$, which is not observed in the real situation (phase lag shows a peak at the transition point). However, this simple picture can anticipate the convex (looking from the origin) nature [6] of the dynamic phase boundary. As the temperature increases $m_{r}$ increases and it also increases as the field amplitude increases. In the simplest asumption, one can consider $m_{r}$ is proportional to the product of $h_{0}$ and $T$. Demanding, $m_{r}=2$ for the dynamic transition $(Q=0)$, one can readily obtain $\left(h_{0}\right)_{d} T_{d}=$ constant. This equation tells that the dynamic phase boundary will be convex. The convex nature of the phase boundary remains invariant even if one assumes that $m_{r}$ is any increasing function of both $T$ and $h_{0}$ (for example, power law; $m_{r} \sim T^{x} h_{0}^{y}$, in this particular case the equation of the dynamic phase boundary becomes $T_{d}^{x}\left(h_{0}\right)_{d}^{y}=$ constant, it is easy to see that this gives the convex shape of the dynamic phase boundary ). However, this very simple asumption can not describe the entire form of the phase boundary accurately, particularly near the end points $\left(\left(h_{0}\right)_{d}=0\right.$ and $\left.T_{d}=0\right)$.

\section{GENERAL RELATION AMONG DYNAMIC ORDER PARAMETER, HYSTERESIS LOOP AREA AND THE DYNAMIC CORRELATION}

From the usual definitions (given in earlier section) of $C$ and $A$, one can write

$$
\frac{1}{\sqrt{2 \pi}}\left(\frac{2 \pi C}{\omega h_{0}}-i \frac{A}{\omega h_{0}}\right)=\frac{1}{\sqrt{2 \pi}} \oint m(t) \exp (-i \omega t) d t,
$$

where $m(\omega)=\frac{1}{\sqrt{2 \pi}} \oint m(t) \exp (-i \omega t) d t$. So,

$C=\frac{h_{0} \omega}{\sqrt{2 \pi}} \operatorname{Re}(m(\omega))$ 
and

$A=-h_{0} \omega \sqrt{2 \pi} \operatorname{Im}(m(\omega))$.

The general (complex) form of $m\left(\omega^{\prime}\right)$ will be

$m\left(\omega^{\prime}\right)=\left|m\left(\omega^{\prime}\right)\right| \exp (i \phi)$

$m\left(\omega^{\prime}\right)=\frac{1}{\sqrt{2 \pi}}\left(\frac{4 \pi^{2} C^{2}}{h_{o}^{2} \omega^{\prime 2}}+\frac{A^{2}}{h_{0}^{2} \omega^{\prime 2}}\right)^{1 / 2} \exp i\left[-\tan ^{-1} \frac{A}{2 \pi C}\right]$

So, $Q$ is related with $A$ and $C$ as follows

$Q=\frac{1}{\tau} \oint m(t) d t=\frac{1}{\sqrt{2 \pi} \tau} \int d \omega^{\prime} \oint m\left(\omega^{\prime}\right) \exp \left(i \omega^{\prime} t\right) d t=\frac{1}{2 \pi \tau} \int d \omega^{\prime} \oint \sqrt{\left(\frac{4 \pi^{2} C^{2}}{h_{o}^{2} \omega^{\prime 2}}+\frac{A^{2}}{h_{0}^{2} \omega^{\prime 2}}\right)} \mathbf{e}^{\left[i\left(\omega^{\prime} t-\tan ^{-1} \frac{A}{2 \pi C}\right)\right]} d t$.

Above equation gives the general relationship among $Q, A$ and $C$.

It has been observed that the steady response $m(t)$, to a sinusoidally oscillating magnetic field $\left(h(t)=h_{0} \cos (\omega t)\right)$, is periodic (with phase lag $\delta$ ) and has the same periodicity $(\tau=2 \pi / \omega)$ of the field. So, one can write $m(t)$ in a Fourier series as

$$
m(t)=a_{0}+\sum_{n=1}^{\infty} a_{n} \cos (n \omega t)+\sum_{n=1}^{\infty} b_{n} \sin (n \omega t) .
$$

From the usual definitions of $Q, A$ and $C$, it is easy to see that

$a_{0}=Q, a_{1}=2 C / h_{0} \quad$ and $\quad b_{1}=A /\left(\pi h_{0}\right)$.

So, one can write

$$
m(t)=Q+\frac{2 C}{h_{0}} \cos (\omega t)+\ldots .+\frac{A}{\pi h_{0}} \sin (\omega t)+\ldots \ldots
$$

Keeping only the first harmonic terms (ignoring higher harmonics) one can easily express the instantaneous magnetization as

$$
m(t)=Q+m_{0} \cos (\omega t-\delta)
$$

where the amplitude of magnetization is $m_{0}=\left[\left(2 C / h_{0}\right)^{2}+\left(A /\left(\pi h_{0}\right)\right)^{2}\right]^{1 / 2}$ and the phase lag is $\delta=\tan ^{-1}(A /(2 \pi C))$.

\section{THE MODEL AND THE SIMULATION SCHEME}

\section{A. The Monte Carlo study}

The local field (at time $t$ ) at any site $i$, of a nearest neighbour ferromagnetic Ising model in the presence of a time varying external magnetic field $h(t)$ with homogeneous and unit interaction energy can be written as

$$
h_{i}(t)=\sum_{j} \sigma_{j}(t)+h(t)
$$

where $\sigma_{i}(t)= \pm 1$ and $j$ runs over the nearest neighbour of site $i$. The local field (at site $i$ ) $h_{i}(t)$ has an external field part $h(t)$, which is oscillating sinusoidally in time

$$
h(t)=h_{0} \sin (2 \pi f t)
$$

where $h_{0}$ and $f$ are the amplitude and frequency of the oscillating field.

According to heat-bath dynamics, the probability $p_{i}(t)$ for the spin $\sigma_{i}(t)$ will be up at time $t$ is given as

$$
p_{i}(t)=\frac{e^{h_{i}(t) / K_{B} T}}{e^{h_{i}(t) / K_{B} T}+e^{-h_{i}(t) / K_{B} T}}
$$


where $K_{B}$ is the Boltzmann constant which has been taken equal to unity for simplicity. It may be noted here that the spin-spin interaction strength $J$ has been taken equal to unity. The temperature $T$ is measured in the unit of $J / K_{B}$. Field is measured in the unit of $J$. The spin $\sigma_{i}(t)$ is oriented (at time $t$ ) as

$$
\sigma_{i}(t+1)=\operatorname{Sign}\left[p_{i}(t)-r_{i}(t)\right]
$$

where $r_{i}(t)$ are independent random fractions drawn from the uniform distribution between 0 and 1 .

In the simulation, a square lattice $(L \times L)$ is considered under periodic boundary conditions. The initial condition is all spins are up (i.e., $\sigma_{i}(t=0)=1$, for all $i$ ). The multispin coding technique is employed here to store 10 spins in a computer word consisting of 32 bits. 10 spins are updated simultaneously (or parallel) by a single command. All words (containing 10 spins) are updated sequentially and one full scan over the entire lattice consists of one Monte Carlo step per spin (MCSS). This is the unit of time in the simulation. The instantaneous magnetisation $\left(m(t)=\left(1 / L^{2}\right) \sum_{i} \sigma_{i}(t)\right)$ is calculated easily. Some transient loops were discarded to have a stable loop and all the dynamical quantities were calculated from the stable loop.

This simulation is performed in a SUN workstation cluster and the computational speed recorded is 7.14 Million updates of spins per second.

\section{B. The Meanfield study}

The meanfield dynamical equation of Ising ferromagnet in the presence of time varying magnetic field is 4 ]

$$
\frac{d m}{d t}=-m+\tanh \left(\frac{m(t)+h(t)}{T}\right),
$$

where the external time varying field $h(t)$ has the previously described sinusoidal form. $T$ is the temperature measured in the unit of $z J / K_{B}$ ( $z$ is coordination number and $K_{B}$ is Boltzmann constant). This equation has been solved for $m(t)$ by fourth order Runge-Kutta method by taking the initial condition $m(t=0)=1.0$. The value of the time differential $(d t)$ was taken $10^{-3}$, so that the error is $O\left(d t^{5}\right) \sim 10^{-15}$. The frequency $\omega$ of the oscillating field is kept fixed $(\omega=2 \pi)$ throughout the study. Some transient loops were discarded and all the values of the response are calculated from a stable loop.

\section{RESULTS}

\section{A. The Monte Carlo results}

In the MC simulation, a square lattice of linear size $L=1000$ is considered. The frequency $\omega$ of the oscillating field has been kept fixed $(\omega=2 \pi \times 0.01)$ throughout the study. From the Monte Carlo simulation technique described above the $m-h$ or hysteresis loops were obtained. Some $(\sim 600)$ initial transient loops were discarded to have the stable loop. From this one can easily estimate the length of the simulation. For the above choice of frequency, 100 MCSS are required to form a complete loop (or cycle), and 600 such loops were discarded. It has been checked carefully that the loop gets stabilised (within a reasonably useful errorbars) for this choice. The dynamic order parameter $Q=\frac{\omega}{2 \pi} \oint m(t) d t$ is readily calculated. The loop area $A$ and the dynamic correlation $C$ have been calculated from the usual definitions. The phase lag $\delta$ (between field and magnetization) has been calculated by taking the difference between the positions of minimum of magnetization and the magnetic field [6]. All values of $Q, A, \delta$ and $C$ for a particular temperature were obtained by averaging over 10 different random samples to obtain the smooth variation. Fig. 1 demonstrates the dynamic transition (with dynamic symmetry breaking) and the related phemomena (e.g., temperature variations of $A, \delta$ etc.) at a glance. For a fixed field amplitude $h_{0}=0.7$ the time variations of $h(t)$ and $m(t)$ are plotted for various temperatures in the pictures in left column and the corresponding $m-h$ loops are shown in the right column. For very low temperature (topmost pictures of Fig. 1), since no spin flip occurs (within the time period) the magnetization $m(t)$ remains constant (unity) and consequently the $m-h$ loop is a straight line having zero loop area. The dynamic order parameter is unity. The concept of phase lag (between $m(t)$ and $h(t))$ is not applicable here. Obviously the dynamic correlation is zero. After slight increase of temperature (pictures in the second row) some small number of spin flips occurs (within the time period). For some time, $m(t)$ decreases from unity and again it becomes equal to unity. The phase lag is the frequency $(\omega)$ times the time difference between the positions of minimum of $m(t)$ and $h(t)$. The $m-h$ loop encloses a finite but small area giving $Q$ less than unity. 
The dynamic correlation starts to grow. As the temperature increases further the phase lag $(\delta)$ and the loop area $A$ increases (pictures in the third row) and the dynamic order parameter $Q$ decreases. In all three cases, described so far, the asymmetric shapes of the $m-h$ loops are observed due to asymmetric time variation of the response magnetisation $m(t)$. The dynamic correlation decreases. As the temperature is very close to the dynamic transition temperature, (fourth row), where the time variation of the response magnetisation is almost symmetric giving maximum values of $\delta$. The $m-h$ loop is symmetric and the dynamic order parameter $Q$ is almost zero. The dynamic correlation $C$ becomes negative and minimal. As one increases the temperaure further (last row), the phase lag decreases, and the loop area decreases. The dynamic correlation starts to grow further. It may be noted here that the conventional hysteresis or $m-h$ loop is observed in this region of temperature. As the temperature increases further the dynamic correlation grows, shows a maxima or peak and then decreases. The loop area monotonically decreases.

The dynamical phase transition, via the dynamical symmetry breaking of the hysteresis loops, has been observed in highly anisotropic and ultrathin (2D Ising like) ferromagnetic films $(\mathrm{Co} / \mathrm{Cu}(001)$ and $\mathrm{Fe} / \mathrm{W}(110))$ 10,11 by using surface magneto optic Kerr effect study at room temperature. In the recent experimental study [11] in ultrathin $\mathrm{Fe} / \mathrm{W}(110)$, the dynamical symmetry breaking of the hysteresis loop was nicely depicted in Fig. 1 of Ref. [11].

The temperature variations of $Q, \delta, C$ and $A$ for two different values of field amplitudes $h_{0}$ are shown in Fig. 2. In both the cases, it has been observed that, near the dynamic transition point $(Q=0)$ the phase lag gives a peak and the dynamic correlation $C$ gives a shallow dip. The dynamic correlation $C$ gives a smeared peak much above (around $T=2.6$ ) the static (ferro-para) transition point $\left(T_{c}=2.269 .\right.$. ) (see Fig. 2). The hysteresis loop area $A$ shows a peak above the dynamic transition point.

It is possible to explain these observations from very simple analytical results described above (section II). The phase lag $\delta$ becomes maximum near the dynamic transition point. So,according to the analytical formulation (for $C$ and $A$ ) for a fixed value of the field amplitude as the temperature increases the loop area $A\left(=4 h_{0} \sin \delta\right)$ starts to increases as the dynamic order parameter $Q$ starts to decrease and above the dynamic transition point (complete spin reversal) the loop area will be maximum and after that $A$ will start to decrease. Similarly, the dynamic correlation $C$ will remain approximately equal to zero until a considerable amount of spin flip occurs and $Q$ changes appreciably and then starts to increase. Above and near the transition point, where the phase lag $\delta$ decreases as temperature increases and $C=\frac{2 h_{0}}{\pi} \cos (\delta)$ should start to increase. Which has been observed indeed. However, near the transition point it gives a shallow dip, where the value of the dynamic correlation $C$ in minimum and negative. The phase lag $\delta$ should be less than or at most equal to $\pi / 2$. The field $\left(h(t)=h_{0} \cos (\omega t)\right)$ crosses zero first at the phase value $\pi / 2$ and it becomes minimum (maximum negative) at the value of phase ( $\omega t)$ equal to $\pi$. The response magnetisation, should change its sign (cross zero) within this period. This is true for $\omega \rightarrow 0$ limit, however for finite but sufficiently high frequency, this will not happen. The phase difference more than $\pi / 2$ would be observed yielding the unconventional shapes of $m-h$ or hysteresis loops. In practice, it was observed that some asymmetric shape of the $(m-h)$ loop gives the value of phase lag $\delta$ slightly higher than $\pi / 2$. In this region, $\cos (\delta=\pi / 2+\epsilon)=-\sin (\epsilon)$, which is negative and will show a shallow dip (cusp like shape) at the point where $\delta$ is maximum. According, to the analytical prediction, the loop area $A\left(=4 h_{0} \sin (\delta)\right)$ should show maximum at the transition point. However, strictly speaking and in practice it has been observed that the loop area $A$ becomes peaked above the transition temperature. Since the loop area is much more strongly dependent on the actual shape of the magnetisation (which is not a perfect square wave in the temperature range concerned here). As the field amplitude increases the transition points shift towards the lower temperature. The maximum of $\delta$ also increases and consequently the dip of $C$ becomes deeper and it remains negative over wider range of temperature (since $\delta$ remains larger than $\pi / 2$ over wider range). It may be noted that the dynamic correlation $C$ becomes zero (in the disordered or $Q=0$ region) where the phase lag $\delta=\pi / 2=1.57080 \ldots$ The dynamic correlation $C$ shows a smeared peak at quite higher temperature (above the Onsager value), which was misinterpreted [12] as a signature of the stochastic resonance. In the MF study (next section), it was shown that this is also present in the absence of fluctuations (or stochasticity).

A similar previous study [6], showed that the ac susceptibility components would give peak (or dip) near the transition point. In that case, the susceptibility components were calculated from the phase lag $\delta$. The phase lag $\delta$ would show a peak at the transition point. As a consequence the susceptibility components would show peak (or dip) reflecting the behaviour of phase lag $\delta$. However, in this case, the three measurements of phase lag $\delta$, loop area $A$ and dynamic correlation $C$ are completely independent, and indicate the transition point separately.

\section{B. The Meanfield results}

By solving the above meanfield equation the $m-h$ or hysteresis loops were obtained. The dynamic order parameter

$Q=\frac{\omega}{2 \pi} \oint m(t) d t$ is readily calculated. The loop area $A$ and the dynamic correlation $C$ have been calculated by using the above definitions. The phase lag (between field and magnetization) has been calculated by taking the difference 
between the minima positions of magnetization and the magnetic field [6]. Fig. 3 shows the temperature variations of $Q, \delta, C$ and $A$ for two different values of field amplitudes $h_{0}$. In both the cases, it has been observed that, near the dynamic transition point $(Q=0)$ the phase lag gives a peak and the dynamic correlation $C$ gives a shallow dip. The hysteretic loss $A$ gives peak above the transition (dynamic) point. The dynamic correlation $C$ gives a smeared peak much above (around $T=1.3$ ) the static (ferro-para) transition point $\left(T_{c}=1.0\right)$ (for a closer view see Fig. 4).

This high temperature peak of the dynamic correlation was misinterpreted as a signature of stochastic resonance [12. This peak is indeed present in the case where the fluctuation is absent (MF case). The appearance of this peak at higher temperature can be explained as follows: for much higher temperature the time variation of instantaneous magnetisation is no longer a square wave like and becomes almost sinusoidal with a phase lage $\delta$. In a very simple view, it can be approximated as $m(t)=m_{0} \cos (\omega t-\delta)$ (from eqn 3.4; $Q=0$ at very high temperature). The dynamic correlation becomes $C=\frac{m_{0} h_{0}}{2} \cos (\delta)$. Where $m_{0}$ is the amplitude of the magnetisation which monotonically decreases as the temperature increases. The phase lag $\delta$ also monotonically decreases in the higher temperature. Consequently $\cos (\delta)$ increases and $m_{0}$ decreases as temperature increases. So, one would obviously expect a peak at a finite temperature (high enough) where the competition, between fall of $m_{0}$ and rise of $\cos (\delta)$ with respect to the temperature $T$, becomes comparable. No stochasticity is involved in it! The loop area $A$ also gives a peak above the transition point. Due to the similar reason given in the earlier section the dynamic correlation $C$ gives a shallow dip near the transition point.

\section{SUMMARY}

The dynamical response of the kinetic Ising model in presence of a sinusoidally oscillating magnetic field has been studied both by Monte Carlo simulation (in two dimension) and by solving the meanfield dynamical equation of motion for the average magnetization.

A general relationship among the hysteresis loop area $A$, dynamic order parameter $Q$ and the dynamic correlation $C$ has been developed (eqn. 3.1). The time series of the magnetization can be decomposed in a Fourior series and the constant term is identifed as the dynamic order parameter $Q$, the amplitudes of first harmonic terms are found to be related to the hysteretic loss (for sine term) and the dynamic correlation (for cosine term) (eqn. 3.3).

The dynamic order parameter, the loop area and the dynamic correlation have been calculated separately (both from MC and MF study) and studied as a function of temperature. It was observed (in both cases) that the dynamic correlation shows shallow (negative) dip near the transition point. The dynamic transition point has been identified as the the minimum-correlation point. The hysteretic loss $A$ becomes maximum above the dynamic transition point. In this sense, the dynamic transition point is not the maximum lossy point. It may be noted that the earlier study [6] of the ac susceptibility suggests that the dynamic transition point would be the maximum-lossy point, since the imaginary part (or lossy- part) of the ac or complex susceptibility also shows a peak near the dynamic transition point. However, there is a remarkable distinction from the present study. In the earlier study [6], the phase lag was calculated from the simulations and the ac susceptibility components were calculated from the phase lag. So, it is expected that the temperature variations of the phase lag will be reflected directly in the temperature variations of ac susceptibility components. But, in the present study the measurements of phase lag, dynamic correlation and the loop area are completely independent. This behaviour of the dynamic correlation is explained from a simple square wave like time variation of the instantaneous response magnetisation. The oversimplified asumption is incapable of explaining the peak position (above the transition point) of the hysteretic loss $A$. However, this simple picture can qualitatively describe the nonmonotonic temperature variations of $A$ and $C$.

The high temperature (above the static critical point $T_{c}$ ) peak of the dynamic correlation was misinterpreted [12] as a signature of the stochastic resonance. This was also discussed and an analytical form of the dynamic correlation was proposed to show that the high temperature peak of the dynamic correlation is present even in the absence of fluctuations (or stochasticity).

Along with the dynamic correlation, the dynamic transition can be identified by various thermodynamic quantities like ac susceptibility [6], relaxation time [8], specific heat [8], susceptibility [9] and the fluctuations of dynamic order parameter and energy [9]. All these quantities indicate the thermodynamic natures of this kind of nonequilibrium dynamic phase transition by showing peak, dip or divergence near the transition point.

The related phenomena of this kind of nonequilibrium dynamic phase transition in the kinetic Ising model are mostly based on observations and not yet analysed by using rigorous theoretical foundations of equilibrium statistical mechanics available so far. The experimental evidences [10,11] are still in the primitive stage. Experimentally, only the dynamic symmetry breaking of the hysteresis loops is observed near the transition point. However, the details study of the nature of the transition, the phase boundary and the associated phenomena (described above) has not yet done experimentally. 


\section{ACKNOWLEDGMENTS}

This work is financially supported by Sonderforschungsbereich 341. The author would like to thank B. K. Chakrabarti and D. Stauffer for careful reading of this manuscript.

+ E-mail:muktish@thp.uni-koeln.de

[1] M. Rao, H. R. Krishnamurthy and R. Pandit, Phys. Rev. B 42, 856 (1990)

[2] P. B. Thomas and D. Dhar, J. Phys. A: Math Gen 26, 3973 (1993)

[3] S. Sengupta, Y. Marathe and S. Puri, Phys. Rev. B 45, 7828 (1992)

[4] T. Tome and M. J. de Oliveira, Phys. Rev. A 41, 4251 (1990)

[5] W. S. Lo and R. A. Pelcovits, Phys. Rev. A 42, 7471 (1990)

[6] M. Acharyya and B. K. Chakrabarti, Phys. Rev. B 52, 6550 (1995); in Annual Reviews of Computational Physics, Vol 1, 107 (1994), Ed: D. Stauffer, (World Scientific, Singapore)

[7] S. W. Sides, R. A. Ramos, P. A. Rikvold and M. A. Novotny, J. Appl. Phys. 81, 5597 (1997); J. Appl. Phys. 79, 6482 (1996)

[8] M. Acharyya, Phys. Rev. E, 56, 2407 (1997); Physica A 235, 469 (1997)

[9] M. Acharyya, Phys. Rev. E 56, 1234 (1997)

[10] Q. Jiang, H. N. Yang and G. C. Wang, Phys. Rev. B 52, 14911 (1995); See also, Y. L. He and G. C. Wang, Phys. Rev. Lett., 70, 2336 (1993)

[11] J. S. Suen and J. L. Erskine, Phys. Rev. Lett., 78, 3567 (1997)

[12] Z. Neda, Phys. Rev. E, 51, 5315 (1995); Phys. Lett. A, 210, 125 (1996)

\section{Figure Captions}

Fig.1. A pictorial demonstration of dynamic transition and associated phemomena. The figures in the left column represents the time variation of $h(t)$ and $m(t)$ for different temperatures and the corresponding $m-h$ loop are shown in right column. Temperature increases from top to bottom. Monte Carlo results for $L=1000, \omega=2 \pi \times 0.01$ and $h_{0}=0.7$.

Fig.2. The Monte Carlo results for the temperature variations of $Q, \delta, C$ and $A$ for two different values of field amplitudes. $Q$ (solid lines,(I) for $h_{0}=0.9$ and (II) for $\left.h_{0}=0.7\right), \delta\left(\times\right.$ for $h_{0}=0.9$ and $\diamond$ for $\left.h_{0}=0.7\right), C\left(\triangle\right.$ for $h_{0}$ $=0.9$ and + for $\left.h_{0}=0.7\right)$ and $A$ ( $\star$ for $h_{0}=0.9$ and $\square$ for $\left.h_{0}=0.7\right)$.

Fig.3. The mean-field results for the temperature variations of $Q, \delta, C$ and $A$ for two different values of field amplitudes. $Q$ (solid lines, (I) for $h_{0}=0.3$ and (II) for $\left.h_{0}=0.2\right), \delta\left(\times\right.$ for $h_{0}=0.3$ and $\diamond$ for $\left.h_{0}=0.2\right), C\left(\triangle\right.$ for $h_{0}$ $=0.3$ and + for $h_{0}=0.2$ ) and $A$ ( $\star$ for $h_{0}=0.3$ and $\square$ for $\left.h_{0}=0.2\right)$.

Fig.4. The closer view of Fig.3. for the dynamic correlation plotted against the temperature (for fixed field amplitude $\left.h_{0}=0.2\right)$. 

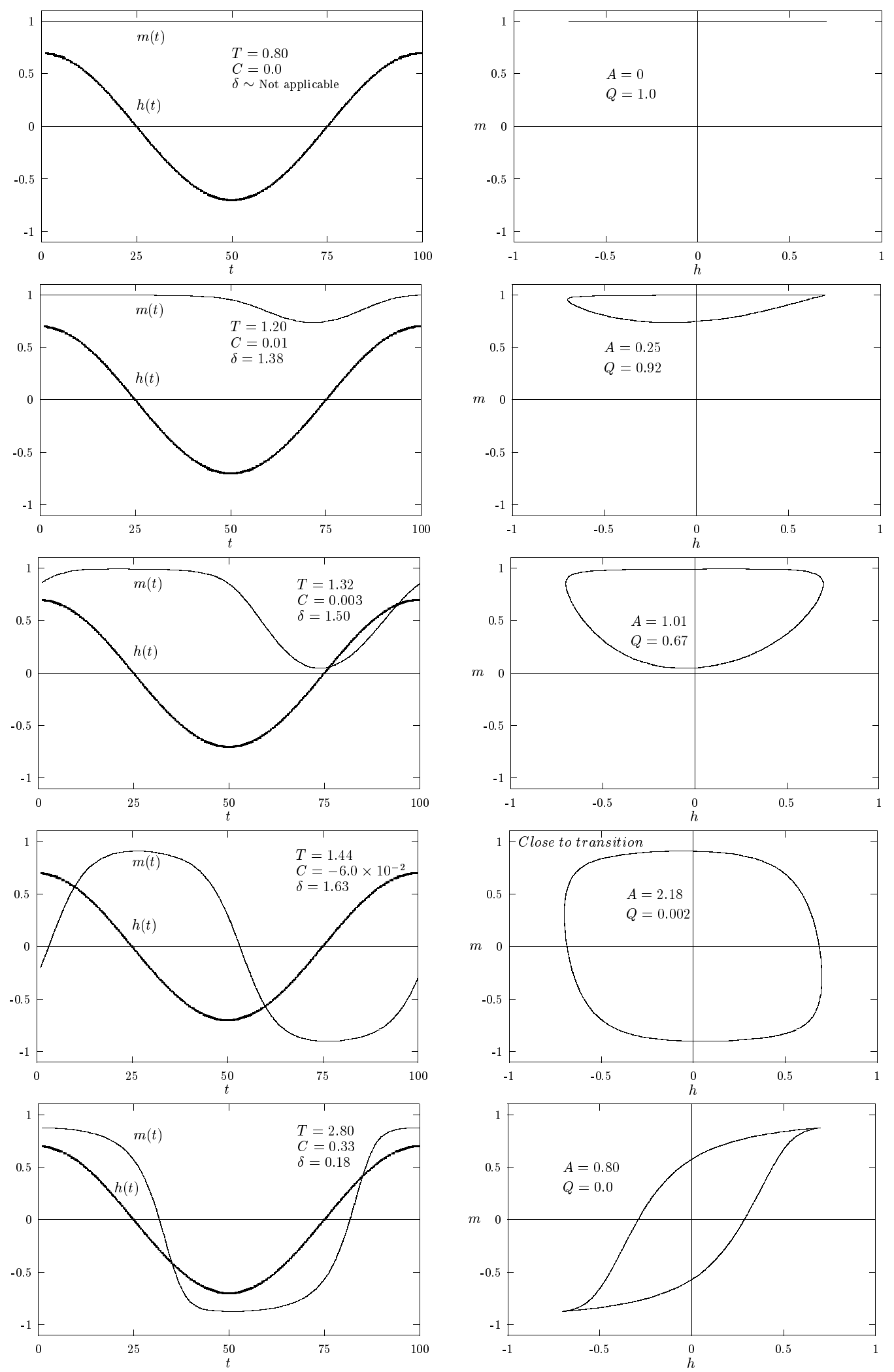
Fig. 2 (PRE, Acharyya)

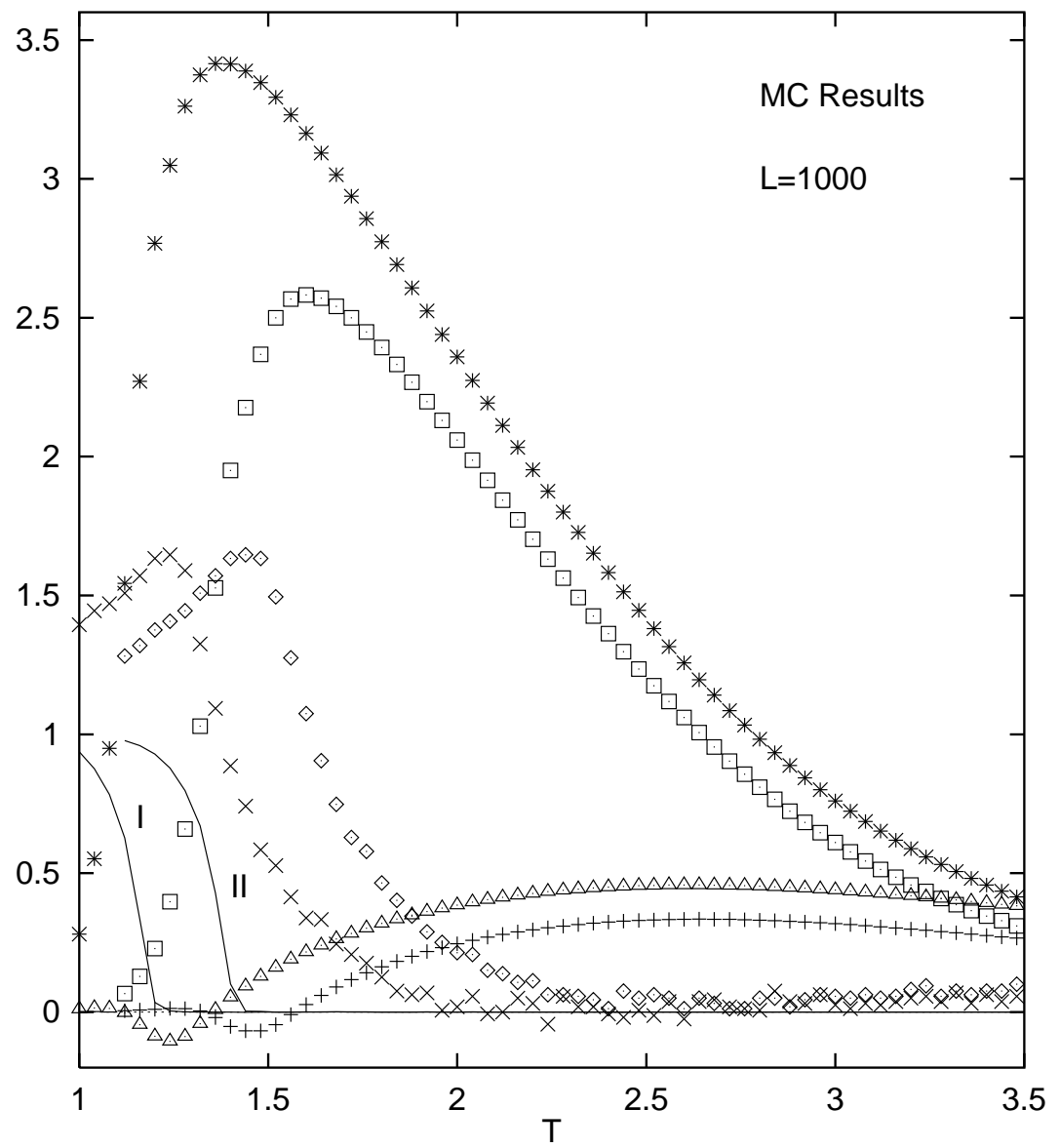


Fig. 3 (PRE, Acharyya)

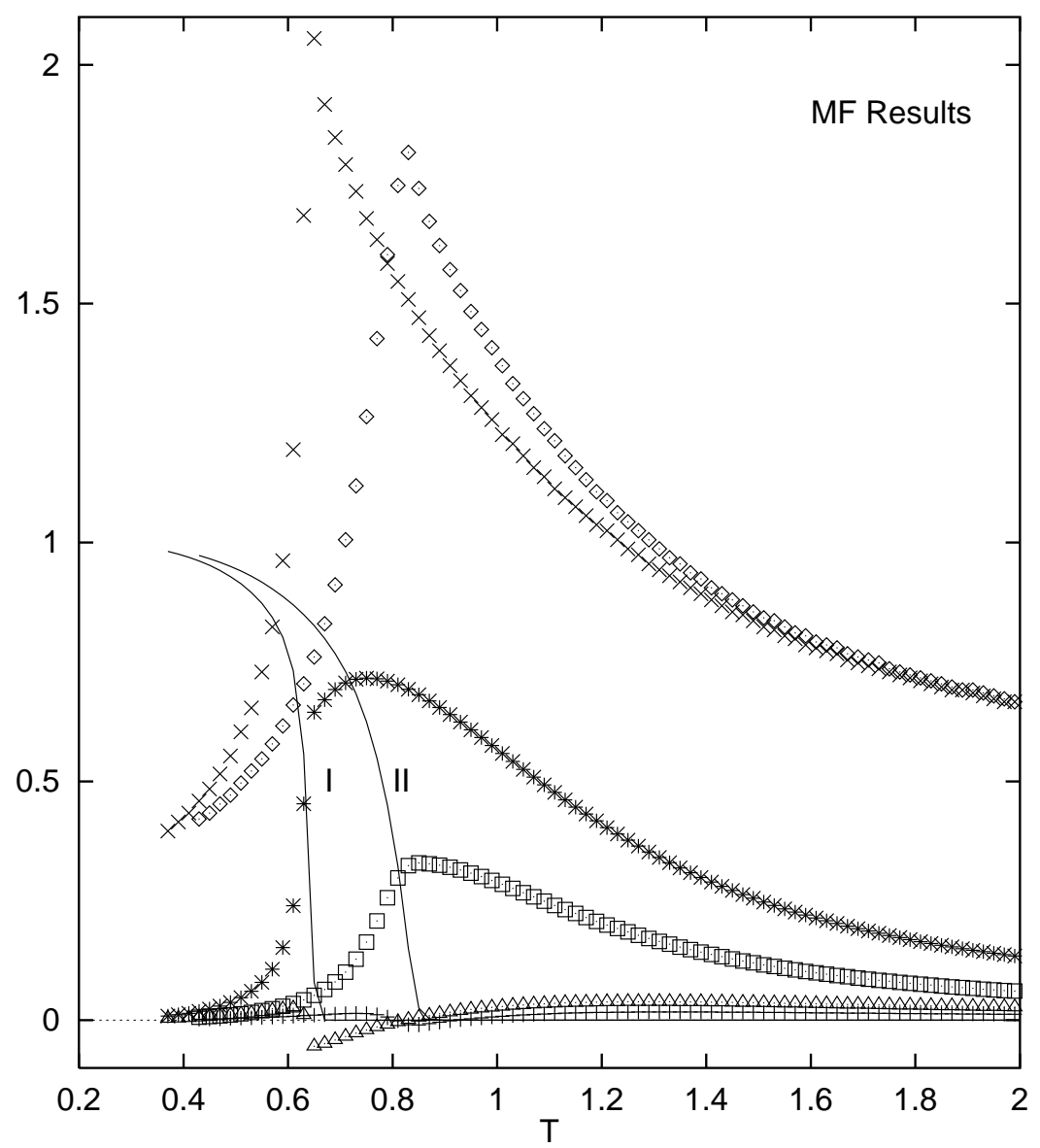


Fig. 4 (PRE, Acharyya)

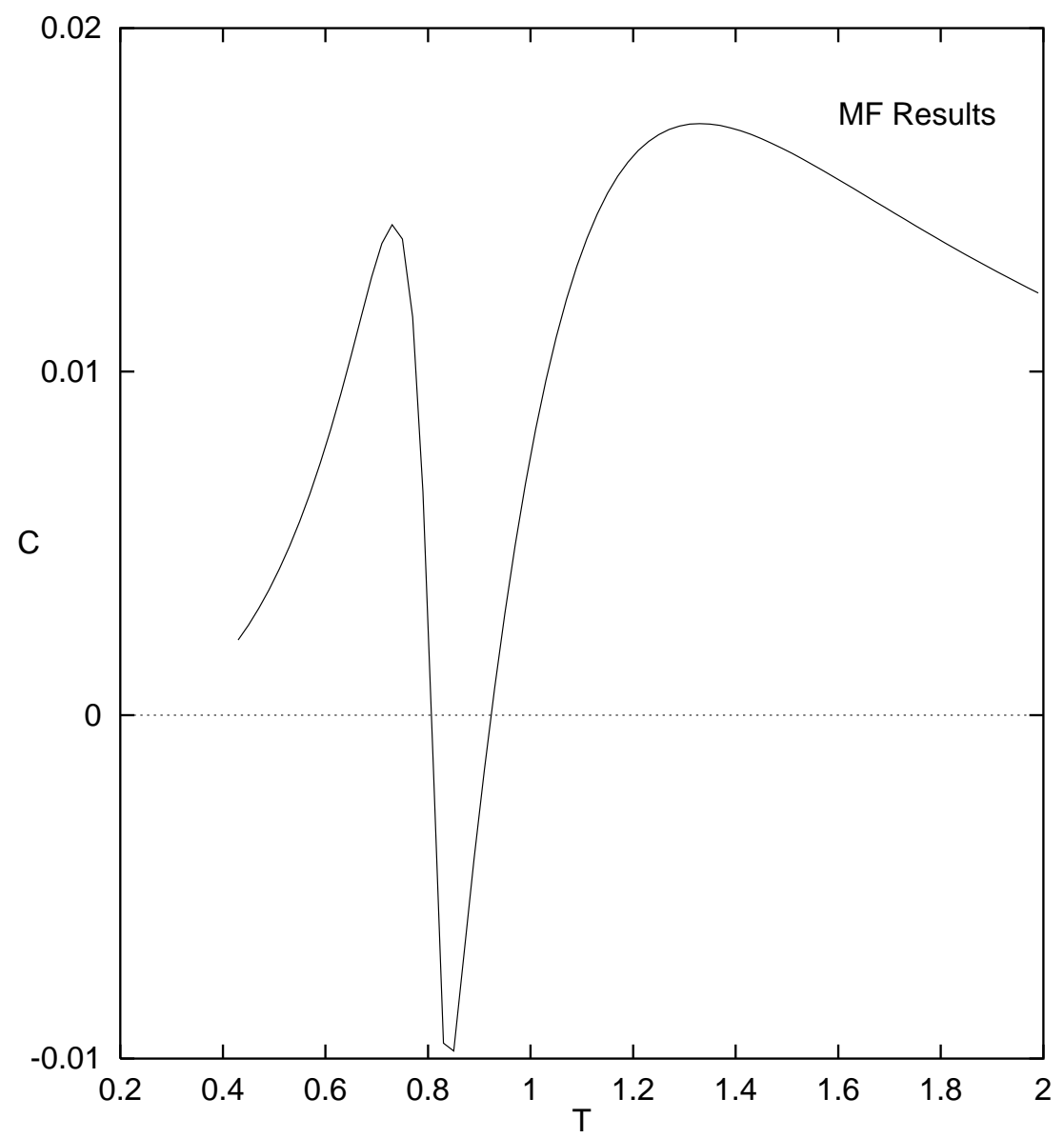

